Proceedings of the Edinburgh Mathematical Society (2002) 45, 723-730 (C)

DOI:10.1017/S0013091501000116 Printed in the United Kingdom

\title{
THE COMPONENT NUMBER OF LINKS FROM GRAPHS
}

\author{
EUNICE GOGO MPHAKO \\ School of Mathematical and Computing Sciences, Victoria University, \\ Wellington, New Zealand (egmphako@unima.mw)
}

(Received 19 January 2001)

\begin{abstract}
This paper gives a number which is used to determine the component number of links from their associated planar graphs. In particular, we use this number to determine the component numbers of links whose associated planar graphs are fans, wheels and 2-sums of graphs.
\end{abstract}

Keywords: component number; knots; wheels; fans; link; 2-sum

AMS 2000 Mathematics subject classification: Primary 05C10

\section{Introduction}

Graphs are currently being used as a research tool in knot theory (see, for example, [4-6]). Every link diagram has an associated planar graph. Thus it is possible to classify knots according to their associated planar graphs and make some generalizations of these classes. In this paper, the term component number will mean the number of components of a link.

For a planar graph $G$, the relationship between the number of crossing cycles of the medial graph of $G$ and the Tutte polynomial of $G$ is well known in the literature (see, for example, $[\mathbf{3}, \mathbf{8}])$. Furthermore, it is known that the medial graph of a planar graph $G$ is a link universe of the associated link of $G$. A link can be obtained from a link universe by defining the part which goes under or over at a crossing. All links obtained in this manner from a single universe have the same component number. Moreover, the component number is the number of crossing cycles of the medial graph. For further details on how to get links from graphs we refer to $[\mathbf{2}, \mathbf{5}]$.

In this paper, $\S 2$ defines a number of a link which will be used to determine the component number of a link. Section 3 gives a generalization of the component numbers of links whose associated planar graphs are fans. Section 4 generalizes the component numbers of links whose associated planar graphs are wheels. Furthermore, it gives results on how the component number of a link changes on the removal of certain edges in a wheel. Finally, $\S 5$ gives the component numbers of links whose associated planar graphs are the 2-sums of graphs and generalizes these results for wheels. 


\section{Determining component numbers of links}

In this section we define a number $\mathcal{L}(K(G))$ of a link $K$.

We start by introducing some notation. We shall use standard notation of graph theory, knot theory and matroid theory and for more details we refer to the books $[\mathbf{9}],[\mathbf{1}]$ and $[\mathbf{7}]$, respectively. Given a graph $G, G^{\prime}$ denotes a graph $G$ with an edge $e$ deleted and $G^{\prime \prime}$ denotes $G$ with an edge $e$ contracted. Let $K$ be a link and $D$ be its connected link diagram in a plane. Then the diagram $D$ divides the plane into several regions. Starting with the unbounded region, we can colour the regions either black or white so that the neighbouring regions never have the same colour. We colour the unbounded region white. Let $G$ be the connected planar graph associated with the link diagram $D$ in which the vertices of $G$ correspond to the black regions of $D$ and the edges of $G$ correspond to the crossings of $D$. To reverse the process, if we are given any planar graph $G$ then we can associate it with a link diagram universe, $U(D)$.

For a graph $G$, with the Tutte polynomial $T(G, x, y)$, it is well known that an evaluation $T(G,-1,-1)=(-1)^{E(G)}(-2)^{c-1}$, where $c$ is the component number of the link whose universe is the medial graph of $G$. We can thus define, for an unoriented link diagram $K(G)$, a number $\mathcal{L}(K(G))=(-1)^{E(G)}(-2)^{c-1}$, where $c$ is the component number of $K(G)$ and $E(G)$ is the edge set of $G$.

Proposition 2.1. Let $G$ be a planar graph. Then

(a) $\mathcal{L}(K(G))=(-2)^{n-1}$ if $G$ is a graph with $n$ vertices and no edges - thus $\mathcal{L}(K(G))=$ 1 if $G$ is a single vertex;

(b) $\mathcal{L}(K(G))=-1$ if $G$ is an isthmus or a loop;

(c) $\mathcal{L}(K(G))=-\mathcal{L}(K(G \backslash e))$ if $e$ is a loop;

(d) $\mathcal{L}(K(G))=-\mathcal{L}(K(G / e))$ if $e$ is an isthmus; and

(e) $\mathcal{L}(K(G))=\mathcal{L}(K(G \backslash e))+\mathcal{L}(K(G / e))$ if $e$ is neither a loop nor an isthmus.

Proof. These follow from the properties of the Tutte polynomials of graphs and the definition of $\mathcal{L}(K(G))$.

The next proposition gives other graph operations which do not affect the component number of the associated link.

Proposition 2.2. Let $G$ be a planar graph. Then

(i) $\mathcal{L}(K(G))=\mathcal{L}(K(G / e / f))$ if $e$ and $f$ are a series pair; and

(ii) $\mathcal{L}(K(G))=\mathcal{L}(K(G \backslash e \backslash f))$ if $e$ and $f$ are a parallel pair. 

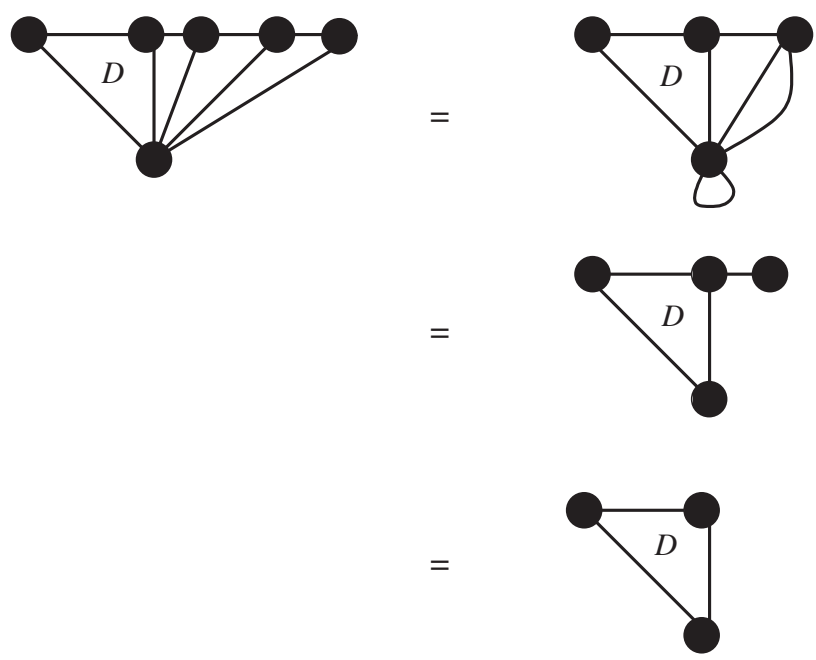

Figure 1.

\section{Component numbers of links associated with fans}

In this section we are going to define a fan and then determine the component number of any link whose associated graph is in this class.

An $n$-fan is a graph with $n+1$ vertices, $\left\{a_{1}, a_{2}, \ldots, a_{n}, x\right\}$, and the set of edges

$$
\left\{\left\{a_{1}, a_{2}\right\},\left\{a_{2}, a_{3}\right\}, \ldots,\left\{a_{n-1}, a_{n}\right\},\left\{a_{1}, x\right\},\left\{a_{2}, x\right\}, \ldots,\left\{a_{n-1}, x\right\},\left\{a_{n}, x\right\}\right\} .
$$

Proposition 3.1. Let $G$ be an $n$-fan. Then $K(G)$ is a 2-link if $n$ is divisible by 3 . Otherwise it is a knot.

Proof. By induction on $n$. If $G$ s a 1 -fan, then $K(G)$ is the 3 -fan and further $K(G)$ is the link $5_{1}^{2}$. Therefore, the proposition is true for $n=1,2,3$. Assume that it is also true for $n=3 m-2,3 m-1$ and $3 m$ for some $m \geqslant 1$. Now consider $n=3 m+1,3 m+2,3 m+3$. In Figure 1 we start with a $d$-fan, $D$ say, and increase it to a $(d+3)$-fan. For simplicity each diagram in the figure represents the number $\mathcal{L}(K(G))$ of the diagram. Thus by applying Propositions 2.2(i), 2.2 (ii), 2.1 (c) and 2.1 (d) accordingly, we get Figure 1.

Hence $\mathcal{L}(K((d+3)$-fan $))=\mathcal{L}(K(d$-fan $))$. Thus substituting $d+3$ by $n$ we have the following results:

$$
\begin{aligned}
& \mathcal{L}(K((3 m+1) \text {-fan }))=\mathcal{L}(K((3 m-2)-\text { fan })), \\
& \mathcal{L}(K((3 m+2) \text {-fan }))=\mathcal{L}(K((3 m-1) \text {-fan })), \\
& \mathcal{L}(K((3 m+3) \text {-fan }))=\mathcal{L}(K((3 m) \text {-fan })) .
\end{aligned}
$$

Therefore, the proposition holds by induction.

\section{Component number of links associated with wheels}

In this section we are going to define a wheel. We will then determine the component number of any link whose associated planar graph is a complete wheel. Finally, we will 

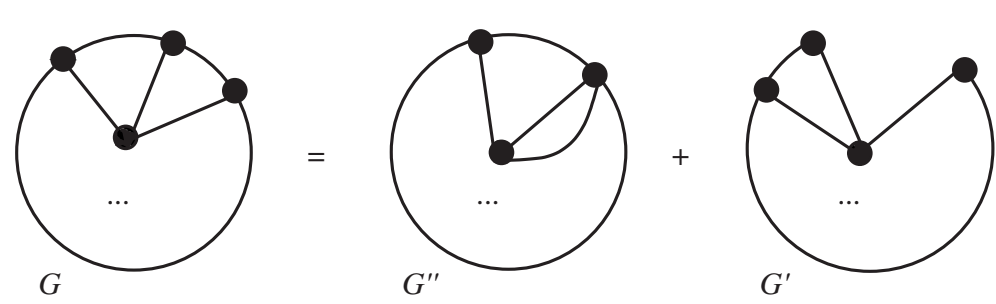

Figure 2.
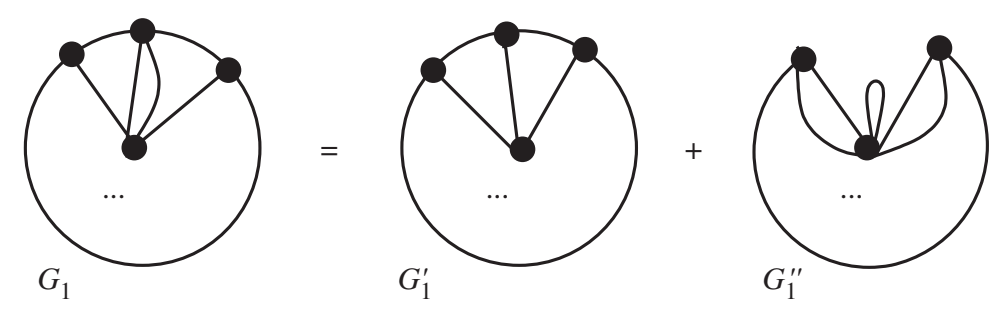

Figure 3 .

determine how the component number of these links changes with the removal of some spokes in the associated planar graph.

An $n$-wheel is a graph with $n+1$ vertices, $\left\{a_{1}, a_{2}, \ldots, a_{n}, x\right\}$, and the set of edges

$$
\left\{\left\{a_{1}, a_{2}\right\},\left\{a_{2}, a_{3}\right\}, \ldots,\left\{a_{n-1}, a_{n}\right\},\left\{a_{n}, a_{1}\right\}\left\{a_{1}, x\right\},\left\{a_{2}, x\right\}, \ldots,\left\{a_{n-1}, x\right\},\left\{a_{n}, x\right\}\right\} .
$$

An edge of the form $\left\{a_{i}, x\right\}$ in an $n$-wheel is called a spoke. An edge of the form $\left\{a_{i}, a_{i+1}\right\}$ for all $i=\{1,2, \ldots, n, n+1=1\}$ in an $n$-wheel is called an arc.

Proposition 4.1. Let $G$ be an $n$-wheel. Then $K(G)$ is a 3-link if $n$ is divisible by 3 . Otherwise it is a knot.

Proof. By induction on $n$. It can be easily verified for $n=1,2,3,4,5,6$. Assume it is true for $n=3 m-2,3 m-1$ and $3 m$ for some $m \geqslant 2$. Now consider $n=3 m+1,3 m+2$ and $3 m+3$. We use diagrams to visualize the computation. For simplicity each diagram in the figures represents the number $\mathcal{L}$ of the link corresponding to the diagram. We start with an $n$-wheel, $G$ say, and we trace the three vertices. Thus by Proposition 2.1 (e) we have the equation represented by Figure 2 .

Thus $G^{\prime}$ is a fan, so we can easily compute $\mathcal{L}\left(K\left(G^{\prime}\right)\right)$ by Proposition 3.1. However, $\mathcal{L}\left(K\left(G^{\prime \prime}\right)\right)$ can be simplified by deleting the parallel pair of edges, followed by contracting the series pair of edges, by Proposition 2.2. We thus get a wheel with three fewer vertices than $G$, but with one pair of parallel spokes, which we call $G_{1}$. Hence $\mathcal{L}\left(K\left(G^{\prime \prime}\right)\right)=$ $\mathcal{L}\left(K\left(G_{1}\right)\right)$. However, by applying Proposition $2.1(\mathrm{e}), \mathcal{L}\left(K\left(G_{1}\right)\right)$ can be simplified as shown in the equation represented by Figure 3.

Thus $G_{1}^{\prime}$ is a wheel with three fewer vertices than $G$. By Propositions 2.1 (c), 2.1 (d) and 2.2 (ii) we can delete the parallel pair, contract the isthmuses and delete the loop of $G_{1}^{\prime \prime}$ getting an $n$-fan with six fewer vertices than $G$. We shall call this fan $G_{2}$. Hence 


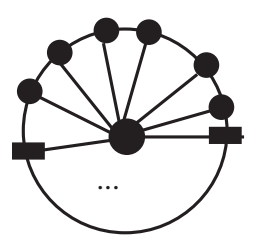

$(d+3)$-wheel

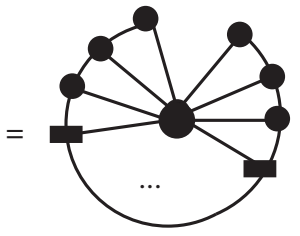

$(d+3)$-fan

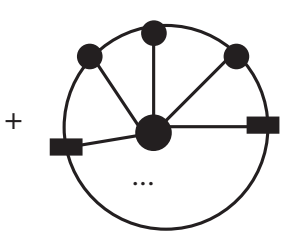

$d$-wheel

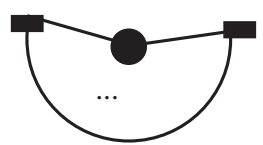

$(d-6)$-fan

Figure 4.

$\mathcal{L}\left(K\left(G_{1}^{\prime \prime}\right)\right)=\mathcal{L}\left(K\left(G_{2}\right)\right)=\mathcal{L}\left(K\left(G^{\prime \prime}\right)\right)$. Thus by substituting back into the equation represented by Figure 2 and letting $G$ be a $(d+3)$-wheel we have the equation represented by Figure 4 .

However, we know that $\mathcal{L}(K((d+3)$-fan $))=\mathcal{L}(K((d-3)$-fan $))$ by Proposition 3.1. Hence $\mathcal{L}(K((d+3)$-wheel $))=\mathcal{L}(K(d$-wheel $))$. Thus for $d=n=3 m+1,3 m+2,3 m+3$ we have

$$
\begin{aligned}
& \mathcal{L}(K((3 m+1) \text {-wheel }))=\mathcal{L}(K((3 m-2) \text {-wheel })), \\
& \mathcal{L}(K((3 m+2) \text {-wheel }))=\mathcal{L}(K((3 m-1) \text {-wheel })), \\
& \mathcal{L}(K((3 m+3) \text {-wheel }))=\mathcal{L}(K((3 m) \text {-wheel })) .
\end{aligned}
$$

Therefore, by induction the proposition holds.

Proposition 4.2. Let $G$ be an $n$-wheel with a missing spoke. Then $K(G)$ is a 2-link.

Proof. We use induction on $n$. The proposition can be easily verified for $n=1,2,3$. Assume it is true for some $k$-wheel with a missing spoke. Now consider $G$ to be a $k+1$ wheel with a missing spoke. By contracting a series pair and throwing away the parallel pair, we get a $k$-wheel with a missing spoke. Therefore, the result is true for any $n$ wheel.

Proposition 4.3. Let $G$ be an $n$-wheel with $q$ consecutive spokes missing.

(a) If $q$ is odd then $K(G)$ is a 2-link.

(b) If $q$ is even and

(i) $q=n-3 k$ for some integer $k \geqslant 1$, then $K(G)$ is a 3-link;

(ii) $q=n-3 k+1$ or $q=n-3 k+2$ for some integer $k \geqslant 1$, then $K(G)$ is a knot.

\section{Proof.}

(a) If $q$ is an odd number, by contracting series pairs we get a wheel with a missing spoke. Hence by Proposition 4.2 it is a 2-link.

(b) If $q$ is an even number, then by contracting series pairs we get an $(n-q)$-wheel. The result follows by applying Proposition 4.1. 


\section{Component numbers of links associated with the 2-sum of graphs}

In this section we are going to define the 2-sum operation for graphs. Then we will determine the component number of any link whose associated planar graph is a 2 -sum of wheels.

Let $G_{1}$ and $G_{2}$ be graphs and let $E\left(G_{1}\right) \cap E\left(G_{2}\right)=e$. The 2-sum of $G_{1}$ and $G_{2}$, denoted by $G_{1} \oplus_{2} G_{2}$, has the edge set $E\left(G_{1}\right) \cup E\left(G_{2}\right) \backslash e$. Here we are only interested with 2-sums which are planar. Let $G_{1}$ be an $n$-wheel and $G_{2}$ be an $m$-wheel. We will call a 2-sum of $G_{1}$ and $G_{2}$ Type A if $e$ is a spoke in $G_{1}$ and $e$ is an arc in $G_{2}$ and Type B if $e$ is an arc in both $G_{1}$ and $G_{2}$. It is clear that 2-sums of wheels of Type A and Type B are planar. The next two propositions are given by Schwärzler and Welsh [8].

Proposition 5.1. The bracket polynomial of a 2-sum of a signed matroid is given by

$$
\begin{aligned}
-\left(A^{4}+1+A^{-4}\right)\left\langle M \oplus_{2} N\right\rangle=\left(A^{2}+A^{-2}\right)\left(\left\langle M_{e}^{\prime}\right\rangle\left\langle N_{e}^{\prime}\right\rangle+\right. & \left.\left\langle M_{e}^{\prime \prime}\right\rangle\left\langle N_{e}^{\prime \prime}\right\rangle\right) \\
& +\left\langle M_{e}^{\prime}\right\rangle\left\langle N_{e}^{\prime \prime}\right\rangle+\left\langle M_{e}^{\prime \prime}\right\rangle\left\langle N_{e}^{\prime}\right\rangle .
\end{aligned}
$$

Proposition 5.2. Let $M$ be the signed cycle matroid of $G$. Then

$$
\langle M, 1\rangle=T(\hat{M},-1,-1),
$$

where $\hat{M}$ is the unsigned version of $M$.

The following proposition is known.

Proposition 5.3. Let $M$ be the cycle matroid of a planar graph $G$. Then

$$
T(M,-1,-1)=T(G,-1,-1)=(-1)^{E(G)}(-2)^{c-1},
$$

where $c$ is the component number of the link whose universe is the medial graph of $G$.

Thus $\langle M, 1\rangle=(-1)^{E(G)}(-2)^{c-1}=\mathcal{L}(K(G))$. Hence we define the number $\mathcal{L}$ of a 2-sum of graphs in the next proposition by applying Proposition 5.1.

Proposition 5.4. Let a planar graph $G_{1} \oplus_{2} G_{2}$ be a 2-sum of planar graphs and $e$ the edge of intersection. Then

$$
\begin{aligned}
-3 \mathcal{L}\left(K\left(G_{1} \oplus_{2} G_{2}\right)\right)=2\left[\mathcal{L}\left(K\left(G_{1}^{\prime}\right)\right) \mathcal{L}(\right. & \left.\left.K\left(G_{2}^{\prime}\right)\right)+\mathcal{L}\left(K\left(G_{1}^{\prime \prime}\right)\right) \mathcal{L}\left(K\left(G_{2}^{\prime \prime}\right)\right)\right] \\
& +\mathcal{L}\left(K\left(G_{1}^{\prime}\right)\right) \mathcal{L}\left(K\left(G_{2}^{\prime \prime}\right)\right)+\mathcal{L}\left(K\left(G_{2}^{\prime}\right)\right) \mathcal{L}\left(K\left(G_{1}^{\prime \prime}\right)\right) .
\end{aligned}
$$

To be able to apply Proposition 5.4 we need to know how the number $\mathcal{L}(K(G))$ changes if we delete or contract $e$ from $G$.

Lemma 5.5. Let $K(G)$ be an $n$-link $n \geqslant 1$. Then $K\left(G^{\prime}\right)$ is an $n$-link if and only if $K\left(G^{\prime \prime}\right)$ is an $(n+1)$-link and vice versa.

Proof. Let $K(G)$ be an $n$-link and $\mathcal{L}(K(G))=2^{n-1}$. Suppose that $K\left(G^{\prime}\right)$ is also an $n$-link. Then $\mathcal{L}\left(K\left(G^{\prime}\right)\right)=-2^{n-1}$. Hence, by Proposition $2.1(\mathrm{e})$,

$$
2^{n-1}+2^{n-1}=\mathcal{L}\left(K\left(G^{\prime \prime}\right)\right)
$$

Thus $\mathcal{L}\left(K\left(G^{\prime \prime}\right)\right)=2^{n}$. Hence $K\left(G^{\prime \prime}\right)$ is an $(n+1)$-link. 
Similarly, if $K\left(G^{\prime \prime}\right)$ is an $(n+1)$-link, we have $\mathcal{L}\left(K\left(G^{\prime}\right)\right)=-2^{n-1}$. Hence $K\left(G^{\prime}\right)$ is an $n$-link.

Lemma 5.6. Let $K(G)$ be an $n$-link for $n \geqslant 2$. Then $K\left(G^{\prime}\right)$ is an $(n-1)$-link if and only if $K\left(G^{\prime \prime}\right)$ is an $(n-1)$-link.

Proof. A similar argument to the proof of Proposition 5.5 gives the result.

Proposition 5.7. Let a planar graph $G$ be a 2-sum of two planar graphs $G_{1}$ and $G_{2}$, each of which corresponds to a link with at least two components. Then $K(G)$ is a link with at least two components.

Proof. By Lemma 5.5 and Lemma 5.6, there are three possible cases of the links corresponding to the minors obtained after deleting or contracting $e$ from $G_{1}$ and $G_{2}$. Let $K\left(G_{1}\right)$ be an $n$-link and $k\left(G_{2}\right)$ be an $m$-link for $n, m>1$. Assume $\mathcal{L}\left(K\left(G_{1}\right)\right)=2^{n-1}$ and $\mathcal{L}\left(K\left(G_{2}\right)\right)=2^{m-1}$.

Case 1. By Lemma 5.6, we can have both $K\left(G_{1}^{\prime}\right)$ and $K\left(G_{1}^{\prime \prime}\right)$ being $(n-1)$-links and both $K\left(G_{2}^{\prime}\right)$ and $K\left(G_{2}^{\prime \prime}\right)$ being $(m-1)$-links. By Proposition $5.4 \mathcal{L}(K(G))=2^{n+m-3}$. Since both $n$ and $m$ are greater than one, then $K(G)$ has at least two components.

Case 2. By Lemma 5.6, we can have both $K\left(G_{1}^{\prime}\right)$ and $K\left(G_{1}^{\prime \prime}\right)$ being $(n-1)$-links. By Lemma 5.5 we can have $K\left(G_{2}^{\prime}\right)$ an $m$-link and $K\left(G_{2}^{\prime \prime}\right)$ an $(m+1)$-link. Hence, by Proposition 5.4, $\mathcal{L}(K(G))=2^{n+m-3}$. Thus $K(G)$ has at least two components.

Case 3. By Lemma 5.5, we can have $K\left(G_{1}^{\prime}\right)$ an $n$-link, $K\left(G_{1}^{\prime \prime}\right)$ an $(n+1)$-link, $K\left(G_{2}^{\prime}\right)$ an $m$-link and $K\left(G_{2}^{\prime \prime}\right)$ an $(m+1)$-link. By Proposition 5.4, $\mathcal{L}(K(G))=2^{n+m-1}$. Since both $n$ and $m$ are greater than one, it follows that $K(G)$ has at least four components.

Corollary 5.8. Let a planar graph $G$ be a 2 -sum of a $(3 n)$-wheel and a $(3 m)$-wheel for any $n, m>1$. Then $K(G)$ is a 4-link.

Proposition 5.9. Let a planar graph $G$ be a 2 -sum of two planar graphs $G_{1}$ and $G_{2}$ such that both $K\left(G_{1}\right)$ and $K\left(G_{2}\right)$ are knots. Then

(i) if $K\left(G_{1}^{\prime}\right)$ and $K\left(G_{2}^{\prime}\right)$ are both knots or links, then $K(G)$ is a 2-link; and

(ii) if $K\left(G_{1}^{\prime}\right)$ is a 2-link and $K\left(G_{2}^{\prime}\right)$ is a knot, then $K(G)$ is a knot.

Proof. Let $\mathcal{L}\left(K\left(G_{1}\right)\right)=\mathcal{L}\left(K\left(G_{2}\right)\right)=2^{0}$.

(i) If $K\left(G_{1}^{\prime}\right)$ and $K\left(G_{2}^{\prime}\right)$ are both knots, then by Lemma $5.5 K\left(G_{1}^{\prime \prime}\right)$ and $K\left(G_{2}^{\prime \prime}\right)$ are both 2 -links and vice versa. Thus by applying Proposition 5.4, $\mathcal{L}(K(G))=-2$. Hence $K(G)$ is a 2 -link.

(ii) If $K\left(G_{1}^{\prime}\right)$ is a 2-link, then by Lemma $5.5, K\left(G_{1}^{\prime \prime}\right)$ is a knot. Similarly, if $K\left(G_{2}^{\prime}\right)$ is a knot, then $K\left(G_{2}^{\prime \prime}\right)$ is a 2-link. Thus by applying Proposition $5.4, \mathcal{L}(K(G))=2^{0}$. Hence $K(G)$ is a knot. 
Corollary 5.10. Let a planar graph $G$ be a 2 -sum of an $n$-wheel and an $m$-wheel, where $n \neq 3 p$ and $m \neq 3 q$ for some integers $p$ and $q$. Then

(i) if $G$ is of Type $A$, then $K(G)$ is a knot; and

(ii) if $G$ is of Type B, then $K(G)$ is a 2-link.

Proposition 5.11. Let a planar graph $G$ be the 2-sum of two graphs $G_{1}$ and $G_{2}$ such that $K\left(G_{1}\right)$ is a knot and $K\left(G_{2}\right)$ is an $n$-link for $n>1$. Then

(a) if $K\left(G_{1}^{\prime}\right)$ is a knot and $K\left(G_{2}^{\prime}\right)$ is an $(n-1)$-link, then $K(G)$ is a $(n-1)$-link;

(b) if $K\left(G_{1}^{\prime}\right)$ is a knot and $K\left(G_{2}^{\prime}\right)$ is an n-link, then $K(G)$ is a $(n+1)$-link;

(c) if $K\left(G_{1}^{\prime}\right)$ is a 2-link and $K\left(G_{2}^{\prime}\right)$ is an $(n-1)$-link, then $K(G)$ is a $(n-1)$-link; and

(d) if $K\left(G_{1}^{\prime}\right)$ is a 2-link and $K\left(G_{2}^{\prime}\right)$ is an n-link, then $K(G)$ is a n-link.

Proof. The proof follows from Proposition 5.4, Lemma 5.5 and Lemma 5.6.

Corollary 5.12. Let a planar graph $G$ be a 2 -sum of a $(3 n)$-wheel and an $m$-wheel such that $m \neq 3 q, q \in \mathbb{Z}^{+}$. Then $K(G)$ is a 2-link.

\section{References}

1. C. C. Adams, The knot book (W. H. Freeman and Company, New York, 1994).

2. B. BollobÁs, Modern graph theory, Graduate Texts in Mathematics, no. 184 (Springer, 1998).

3. F. JAEGER, On transition polynomials of 4-regular graphs, in Cycles and rays, pp. 123-150 (Kluwer, Dordrecht, 1990).

4. L. H. Kauffman, New invariants in the theory of knots, Am. Math. Mon. 95 (1988), 195-242.

5. L. H. Kauffman, A Tutte polynomial for signed graphs, Discrete Appl. Math. 25 (1989), $105-127$.

6. K. Murasugi, On invariants of graphs with applications to knot theory, Trans. Am. Math. Soc. 314 (1989), 1-49.

7. J. G. OXLeY, Matroid theory (Oxford University Press, New York, 1992).

8. W. Schwärzler and D. J. A. Welsh, Knots, matroids and the Ising model, Math. Proc. Camb. Phil. Soc. 113 (1993), 107-139.

9. R. J. WILSON, Introduction to graph theory (Oliver \& Boyd, Edinburgh, 1972). 